\title{
Pró-Saúde e os Quarenta Anos de Integração Docente-assistencial da Faculdade de Odontologia de Piracicaba - Unicamp
}

\author{
The Educational Program for Health Work \\ (Pró-Saúde) and 40 Years of Integration \\ between Medical Education and Healthcare \\ at the Piracicaba School of Dentistry, \\ University of Campinas
}

Marcelo de Castro Meneghim Antonio Carlos Pereira Fábio Luiz Mialhel

Maria da Luz Rosário de Sousa

\section{PALAVRAS-CHAVE \\ - Odontologia em Saúde Pública: \\ - Programa Saúde da Família; \\ - Educação em saúde bucal}

KEYWORDS: PUBLIC

- Health Dentistry;

- Family Health Program;

- Dental Health Education

\begin{abstract}
Recebido em: 01/10/2010
\end{abstract}
Aprovado em: 22/11/2010

REVISTA BRASILEIRA DE EDUCAÇ̃̃o MÉDICA

\section{RESUMO}

Para formar recursos humanos adequadamente preparados para trabalhar no SUS, é importante uma mudança qualitativa no ensino de graduação. Identificada com esses pressupostos, a FOP vem-se empenhado em construir um novo modelo de ensino voltado a atender às demandas de formação. Este artigo tem por objetivo apresentar a experiência da FOP na integração docente-assistencial. No primeiro ano da graduação, os alunos têm contato com a Atenção Básica e Secundária por meio de visitas guiadas. No segundo ano, prioriza-se as formas de promoção e prevenção das principais doenças em Odontologia e, no terceiro ano, são abordados temas de saúde coletiva. No último ano do curso, os alunos fazem seu estágio supervisionado em uma das seis USFs. Essa experiência é realizada em 64 horas divididas nos dois semestres. O programa é desenvolvido de forma integral (8 horas/dia) em quatro dias consecutivos em uma mesma semana. Pode-se concluir que essa atividade possibilita a experiência de praticar os conhecimentos auxiliando significativamente na formação profissional, na dinâmica do trabalho, na interação com a pós-graduação, além da quebra de preconceitos relativos ao serviço.

\section{ABSTRACT}

A qualitative change in undergraduate education is important for adequately training human resources to work in the Unified National Health System. Based on this premise, the Piracicaba School of Dentistry, University of Campinas, has focused on developing a teaching model to meet such training requirements. This article aims to present the experience at the School of Dentistry in integration teaching and patient care. During their first year of undergraduate training, students have contact with primary and secondary care through guided visits. The second year prioritizes health promotion and the prevention of the principal oral and dental problems encountered in dentistry, while the third year focuses on public health issues. In the last year of the course, students participate in a supervised internship in one of the six Family Health Units. This experience covers 64 hours, divided into two semesters. The program is full-time (8 hours/day) on four consecutive days in a single week. The activity allows the experience of practicing knowledge, significantly improving the students' professional training in the work dynamics and interaction with graduate studies, in addition to challenging preconceived ideas about the health services. 


\section{INTRODUÇÃO}

A partir da Constituição de 1988, com a criação do Sistema Único de Saúde (SUS) e sua legitimação no plano jurídico-institucional, o Ministério da Saúde tem trabalhado na tentativa de reestruturar o modelo de atenção no Brasil, na expectativa de reorganizar os serviços da saúde oferecidos à população de modo que estes sejam cada vez mais resolutivos, integrais e humanizados ${ }^{1,2}$. Desde então, a atenção básica passou a ser uma área de concentração de esforços e investimentos e, atualmente, o PSF (Programa de Saúde da Família) é considerado estratégia prioritária para sua organização. Entretanto, para além de um "programa", que designa uma atividade temporária, ou seja, com prazo para início, desenvolvimento e finalização das atividades, o PSF é definido muito mais como uma estratégia, surgindo, assim, o termo Estratégia Saúde da Família(ESF) em substituição ao anterior.

A partir de então tem sido proposto um redirecionamento no processo de trabalho, em que a equipe de saúde da família composta por uma equipe multiprofissional, passa a direcionar suas atividades para a resolução de problemas sociais e de saúde de determinada população adscrita, buscando não apenas oferecer o tratamento curativo, mas também ações voltadas à promoção da saúde e à prevenção de doenças.

Sentindo a necessidade de ampliar o acesso da população brasileira às ações de saúde bucal, o Ministério da Saúde lança a portaria GM/MS no 1.444 , de 28 de dezembro de $2000^{3}$, a qual regulamenta e estabelece incentivo financeiro para a reorganização da atenção à saúde bucal prestada nos municípios. A partir desse momento, as equipes de saúde bucal (ESB) também passaram a fazer parte da estratégia do PSF.

Contudo, as principais dificuldades enfrentadas na construção das políticas de saúde bucal no âmbito da saúde da família têm sido ampliar o quadro de profissionais que se ocupam do cuidado em saúde bucal e repensar o alcance dessa prática, visto que a equipe de saúde bucal não pode ser considerada um apêndice do $\mathrm{PSF}^{4}$. Assim, a atenção à saúde bucal deve ser constituída pelo conjunto de ações que buscam atingir grupos populacionais por meio de um trabalho de alcance coletivo, ou seja, o profissional deve atuar não apenas no âmbito da Odontologia, mas no próprio setor da saúde. Em outras palavras: as ações desenvolvidas em Odontologia devem abranger o conjunto da sociedade, promovendo saneamento, educação, promoção de saúde, etc. ${ }^{5}$

Dessa forma, percebe-se que a formação de recursos humanos adequadamente preparados para trabalhar nesse mercado de trabalho faz-se necessária. Para isso, é importante garantir uma mudança qualitativa no ensino de graduação em que se contemplem as necessidades de atuação no SUS, ou seja, um modelo que extrapole a simples "odontotécnica"6. Identificada com esses pressupostos, a Faculdade de Odontologia de Piracicaba (FOP-Unicamp), sob coordenação do Departamento de Odontologia Social, vem-se empenhado em construir um novo modelo de ensino e extensão voltado a atender às demandas de formação de um profissional capacitado para atuar nos serviços públicos.

Interessante notar que a experiência da FOP-Unicamp nesse campo de formação e em estágios extramuros não é recente. Por essa razão é digno de consideração resgatar esta experiência de aproximadamente quarenta anos em programas de integração docente-assistencial, com a finalidade de apoiar as transformações necessárias em outras instituições. A experiência iniciou-se em 1970, logo após a assinatura, em 1969, de um convênio com a Prefeitura Municipal de Piracicaba/ SP para atendimento odontológico da zona rural do município. Em 1975, foi iniciado um segundo programa de integração docente-assistencial na cidade de Paulínia, estado de São Paulo, que fica a60 km de Piracicaba. Esse programa foi reformulado em 1980 e, posteriormente, em 1983, quando surgiu o Programa Integrado de Educação e Saúde Escolar (Piese), que passou ainda em 1985 por novas alterações, com modificações também no equipamento simplificado utilizado ${ }^{7,8}$.

Em 1989, foi assinado o convênio entre a Prefeitura Municipal de Piracicaba e a FOP, que criou o estágio extramuros na cidade. O programa de estágio extramuros passou por uma nova reformulação e ampliação no ano de 2002, com a inserção de um novo parceiro do setor privado: a Fundação Arcelor Mittal Brasil. Apesar da preocupação da FOP nesse período em formar um profissional sensível às questões do setor público de saúde como um todo, sua abrangência acabou ficando restrita ao âmbito da assistência odontológica.

No final do ano de 2005, por meio de uma iniciativa conjunta dos Ministérios da Saúde e da Educação, foi instituído o programa Pró-Saúde, que, entre outros objetivos, destina-se à reorientação do processo de formação do aluno de graduação, de modo a responder às necessidades da população brasileira e a operacionalização do SUS, por meio da abordagem integral do processo saúde-doença9 .

A FOP submeteu e teve aprovado o seu projeto Pró-Saúde/FOP, tendo como uma das principais diretrizes a inserção do corpo docente e discente na elaboração do programa e do planejamento de atividades das equipes de saúde da família.A partir disso, iniciou-se uma nova etapa com a ampliação do tempo de trabalho no setor público e o aumento da diferenciação dos cenários de prática para os alunos.

Cabe ressaltar que a FOP também estruturou com a Prefeitura Municipal um CEO (Centro de Especialidades Odon- 
tológicas), com o objetivo de implantar a rede de referência e contrar referência, abordando atenção integral ao paciente atendido nas USFs e oferecendo aos alunos de graduação e de pós-graduação, não somente da área de Odontologia em Saúde Coletiva, a oportunidade de conhecer e vivenciar a experiência, como também o desenvolvimento, de pesquisa para o SUS.

A partir do exposto, esta discussão não tem por objetivo propor um método inovador no sistema de trabalho ou realizar uma revisão da literatura sobre os sistemas vigentes, mas busca apresentar a experiência que se narra como exemplo e referencial de discussão. A partir do relato dessa experiência, acredita-se estar contribuindo para a formação de profissionais capazes de desempenhar adequadamente seu papel no âmbito do SUS, com comprometimento social e maior participação em atividades globais integradas às estruturas acadêmicas.

\section{A Caracterização do Município de Piracicaba}

Piracicaba situa-se no interior do estado de São Paulo e sua população estimada em 2008 era de 365.440 habitantes, sendo $96 \%$ dela concentrada na área urbana. A expectativa de vida no município é de 72,95 anos; a taxa de alfabetização é de $94,95 \%$ e o Índice de Desenvolvimento Humano (IDH-M) é de 0,83610 .

Na cidade, a implantação da ESF iniciou-se em 1999 com a construção de cinco unidades. Em 2009,o município contava com 79 estabelecimentos públicos de saúde, sendo nove estaduais e 70 municipais. A Atenção Básica no município é dividida em Unidades Básicas de Saúde (UBSs) e Unidades de Saúde da Família (USFs). As USFs estão localizadas estrategicamente em áreas de maior vulnerabilidade social do município, cuja necessidade de direcionamento dos recursos de saúde é maior.

\section{O Projeto Pró-Saúde da FOP-Unicamp}

O Pró-Saúde, segundo a proposta apresentada pela portaria interministerial, "tem a perspectiva de que os processos de reorientação da formação ocorram simultaneamente em diferentes eixos, em direção apontada pela IES (Instituição de Ensino Superior), que antevê uma escola integrada ao serviço público de saúde, na formação de recursos humanos e na produção do conhecimento, com o objetivo de fortalecer o SUS"9Assim, a primeira ação concreta relativa ao projeto foi a análise institucional (situação desejada) para o planejamento (estratégias propostas) das adequações necessárias, formadas por vetores, como mostrado na sequência:

\section{Vetor 1}

Situação desejada:

- inserir o discente dentro do contexto de atuação das Equipes de Saúde da Família (ESF);

- diminuir a prevalência de problemas da cavidade bucal com a utilização de RH vinculado ao convênio do IES/ gestores do SUS;

- capacitar o graduando de Odontologia para o planejamento das ações de organização da prática odontológica, com base epidemiológica e utilização de critérios de risco para os agravos em saúde bucal da sua população adscrita;capacitar o graduando de Odontologia em relação aosconceitos relativos à humanização e acolhimento;

- capacitar o graduando de Odontologia no que diz respeito a conceitos relativos ao atendimento domiciliar em saúde bucal, de forma a identificar os casos que efetivamente necessitem de assistência domiciliar de acordo com o grau de incapacidade funcional e os limites de atuação no domicílio.

Estratégias para se alcançar a situação desejada:

- visita à USF e a outros órgãos ligados ao SUS;

- inserção do discente na elaboração da programação e planejamento de atividades das Equipes de Saúde da Família;

- visitas domiciliares com utilização de determinantes sociais e diagnóstico biológico para a determinação do prognóstico de tratamento.

- atividades de educação para a saúde e aplicação de métodos de prevenção em espaços sociais (USFs, escolas, creches, outros); desenvolvimento de projetos pelos alunos de pós-graduação vinculados àUSF;

- interação com a disciplina de educação para a saúde (graduação e pós-graduação);

- atividades clínicas de atenção primária e secundária. Nessas atividades, o discente conta com a supervisão de um tutor, que pode ser um profissional do órgão gestor.

\section{Vetor 2}

Situação desejada: Uma clara necessidade de se oferecer cursos de capacitação para os cirurgiões - dentistas dos órgãos gestores do SUS de Piracicaba e região e inserir alguns desses profissionais em cursos de pós-graduação (preferencialmente o mestrado profissional), para melhorar o seu perfil crítico em relação a planejamento e políticas de saúde.

Estratégias para se alcançar a situação desejada: 
- cursos modulares de capacitação para os cirurgiões-dentistas, capacitando-os a trabalhar em equipe multiprofissional dentro do contexto de atuação do SUS;

- curso de capacitação - curso básico de epidemiologia e principais problemas de saúde oral para os membros da equipe saúde da família, com carga horária total de 32 horas, com finalidade de orientá-los para o trabalho multiprofissional, bem como contextualizar o trabalho da FOP junto ao Pró-Saúde, proporcionando condições de integração do serviço com a Universidade.

\section{Vetor 3}

Situação desejada: AIES deveria ter um vínculo mais estreito com os órgãos gestores do SUS, buscando capacitar recursos humanos de uma forma mais compatível com os fundamentos que norteiam o sistema.

Estratégias para se alcançar a situação desejada:

- Oferta de vagas para cirurgiões - dentistas e outros profissionais do serviço para a pós-graduação(mestrado profissional), buscando capacitar recursos humanos com visão crítica e conhecimento mais aprofundado de práticas de planejamento e políticas de saúde.

- Capacitar ACDs e THDsdentro do sistema prestador, buscando uma maior sintonia entre o serviço e a IES.

\section{Vetor 4}

Situação desejada: A época atual é marcada pelas transformações epidemiológicas e demográficas que, aliadas à tendência de aumento na oferta de emprego para o cirurgião-dentista no setor público e à restrição de demanda no setor privado, tem exigido que o profissional da área odontológica tenha competência para organizar o serviço diante dessa nova realidade, segundo as diretrizes do SUS.

Estratégias para se alcançar a situação desejada:

- aumentar a carga horária do aluno nos primeiros anos do curso para estágio supervisionado em Unidades de Saúde da Família;

- inserção de conteúdos sobre aspectos dasaúde e sociedade nos primeiros anos do curso de graduação; participação dos docentes de áreas social e clínica nos estágios supervisionados, para adequar o conteúdo programático aos princípios do SUS.

\section{Vetor 5}

Situação desejada: O desejável seria desenvolver estágio clínico na rede de atenção básica do SUS, com graus de complexidade da atenção básica à terciária, entendendo o planejamento segundo as necessidades do SUS.
Estratégia para se alcançar a situação desejada:

- o desejável seria a criação de uma carga horária para desenvolvimento de atividades extramuros em unidades do SUS, em todos os níveis da atenção.

\section{Vetor 6}

Situação desejada: Integrar ainda mais o serviço da IES com o SUS, principalmente no desenvolvimento dos mecanismos de referência e contrarreferência com a rede do SUS.

Estratégia para se alcançar a situação desejada:

- organização do sistema de referência e contrarreferência com a participação dos professores da Universidade, criando um sistema de fluxo contínuo de pacientes.

\section{Vetor 7}

Situação desejada: Aparticipação do aluno nessas atividades torná-lo-á apto a fazer a análise crítica do serviço, visando ao aperfeiçoamento deste.

\section{Vetor 8}

Situação desejada: Aperfeiçoar o método orientador da integração, acrescentando a um maior número de disciplinas a utilização da problematização como estratégia de aprendizagem.

\section{Vetor 9}

Situação desejada: Desenvolver o ensino com base na problematização e com atividades realizadas em pequenos grupos.

Estratégias para se alcançar a situação desejada:

- promover a capacitação docente na utilização desse método de ensino;

- adequar a metodologia de ensino das disciplinas;

- avaliar o processo.

\section{Proposta de Avaliação do Processo}

A auto avaliação do programa Pró-Saúde da FOP/Unicamp aborda a avaliação em seus diversos aspectos e, portanto, será utilizado o método de Avaliação $360^{\circ}$ adaptado.

Esse método avaliativo consiste em um processo dinâmico que envolve todas as pessoas que participam do processo (alunos, docentes, profissionais da rede e usuários), permitindo informações abrangentes sob diversos ângulos. Assim, os seguintes indicadores são avaliados:

1. Pelos alunos: Os graduandos avaliam o estágio por meio da experiência vivenciada na Unidade e do impacto na sua formação profissional. Os profissionais avaliam o envolvimento, a postura e o comportamento dos alunos nas atividades desenvolvidas na USF e com a população. Os docentes avaliam a interação do aluno com a equipe e usuários da USF. 
Avaliação:

- Contribuição do programa para sua formação profissional.

- Contribuição do programa para sua formação pessoal.

— Estímulo/motivação à prática odontológica.

- Hábitos de trabalho.

- Comunicação e relacionamento com o paciente.

- Prática inovadora desenvolvida.

— Interação com a equipe de saúde.

- Relação docente/profissional da rede.

- Avaliação de pares (como você observa os demais colegas nas atividades).

- Postura crítica em relação ao SUS.

- Adequação da estrutura física.

- Aspectos positivos.

- Aspectos negativos.

2. Pelos docentes/instituição: Toda USF possui um docente responsável pelos graduandos, pelos pós-graduandos e pelas atividades desenvolvidas por eles. $\mathrm{O}$ docente avalia os alunos por meio da análise dos seguintes itens: desempenho do aluno; interação com a equipe de saúde; cooperação nas atividades realizadas; relacionamento com os profissionais da unidade, com as atividades programadas;postura ética em relação ao trabalho e à população; postura crítica em relação ao SUS; apoio e motivação para participação das atividades da USF; aspectos positivos e negativos.

Além de avaliar os alunos, os docentes avaliam o desenvolvimento do estágio a partirde reuniões, durante o semestre, com os graduandos e pós-graduandos. O objetivo dessas reuniões é verificar o andamento das atividades nas USFs e as possíveis alterações que podem ser realizadas durante o ano para que as atividades se tornem mais interessantes e significativas para os diversos processos de aprendizagem.

\section{Avaliação:}

— Interação com a equipe de saúde.

- Postura crítica em relação ao SUS.

- Adequação da estrutura física.

— Cooperação nas atividades realizadas.

- Relacionamento com os profissionais da rede.

- Postura ética da equipe de trabalho.

- Desempenho do aluno.

- Envolvimento da equipe de saúde com as atividades programadas.

- Adequações realizadas no currículo pleno do curso de Odontologia.

— Apoio/motivação à participação no programa.

- Aspectos positivos e negativos.
3. Pelos profissionais da rede: Os profissionais da USF (enfermeira, médico e cirurgião-dentista) analisam o desenvolvimento do aluno de graduação. São avaliados: a contribuição das atividades da USF; a cooperação às atividades realizadas;o apoio e a motivação para participar das atividades da USF; a postura ética diante da equipe e da população; a postura crítica em relação ao SUS; os aspectos positivos e negativos.

Avaliação:

- Relacionamento com os docentes e alunos.

- Adequação da estrutura física.

- Contribuição do programa para sua formação profissional.

- Desempenho do aluno.

- Participação/interação do corpo docente.

- Cooperação nas atividades realizadas.

- Apoio/motivação à participação no programa.

- Postura ética da equipe de trabalho.

- Postura crítica em relação ao SUS.

- Aspectos positivos.

- Aspectos negativos.

4. Pelos usuários: A avaliação é realizada pelos agentes comunitários de saúde (ACS), observando o comportamento do aluno durante as visitas domiciliares, a qualidade das informações prestadas pelo aluno durante a visita e o grau de envolvimento dele nas atividades da USF.

Avaliação:

— Nível de satisfação em relação ao atendimento.

— Comunicação e relacionamento com os profissionais.

- Estrutura física.

— Orientação à Saúde Bucal.

- Acolhimento do serviço.

Os indicadores descritos procuram abranger os eixos teóricos, cenários de prática e pedagógico previstos no programa. No decorrer das atividades, eles podem ser alterados de acordo com as necessidades apresentadas e/ ou sugeridas pelos envolvidos. As informações obtidas nas avaliações são analisadas e discutidas na Comissão de Gestão e Acompanhamento Local e na Comissão de Ensino de Graduação da FOP.

\section{Sistema de Informação}

A FOP optou por trabalhar o sistema de informação a partir de uma plataforma digital (http: //www.ead.unicamp. br/ teleduc) e registro de dados em planilhas eletrônicas e cadernos-ata, estes últimos presentes nas Unidades de Saúde da Família. Os graduandos preenchem o diário de bordo em um sistema informatizado da FOP-Unicamp, chamado Tele- 
duc11durante a semana que frequentam a USF. Esse sistema é um ciberespaço onde são anotadas as atividades realizadas diariamente, nos períodos da manhã e da tarde.

A anotação no caderno-ata impresso é feita diariamente e após o final de cada período (manhã e tarde), evitando, assim, possíveis esquecimentos por parte dos acadêmicos do que ocorreu naquele dia durante as atividades. As anotações no Teleduc possuem como prazo máximo de postagem o final da manhã da segunda-feira posterior ao término do estágio. A utilização do caderno impresso serviu de importante ferramenta durante o período de adaptação ao Teleduc, pois mostrou-se ser uma ferramenta rápida e de fácil acesso para que todos os componentes da equipe pudessem registrar suas percepções.

No Teleduc, os alunos avaliam os seguintes aspectos: contribuição das atividades na USF para sua formação profissional e pessoal; estímulo/motivação à prática odontológica; hábitos de trabalho; comunicação e relacionamento com o paciente; prática inovadora desenvolvida; interação com a equipe de saúde; avaliação de pares (já queo aluno observa os demais colegas nas atividades); postura crítica em relação ao SUS; adequação da estrutura física; aspectos positivos e negativos.

A referência e a contrarreferência segue o sistema implantado pelo município e sua fiscalização fica sob responsabilidade do coordenador gestor local.

\section{Metodologia de Trabalho}

Desde o primeiro ano da graduação os alunos já começam a ter contato com a rede municipal de saúde. Inicialmente, conhecem alguns tipos de serviço oferecidos pelo município, que abrangem a Atenção Básica (UBSs, USFs) e a Atenção Secundária (Pronto-Atendimento, Centro de Especialidades e Policlínica). Nesse momento, os alunos são sensibilizados sobre o papel do cirurgião-dentista no contexto do sistema público de saúde.

Durante o segundo ano, prioriza-se as formas de promoção e prevenção das principais doenças em Odontologia e, no terceiro ano, é desenvolvida a disciplina de Odontologia Preventiva e Saúde Pública, nas quais são abordados assuntos como: levantamentos epidemiológicos, políticas públicas em saúde, sistemas de trabalho, financiamento do setor saúde, planejamento em saúde e vigilância epidemiológica e sanitária.

No último ano do curso, os alunos fazem seu estágio supervisionado em uma das seis USFs do município que compõem o programa de estágio extramuros. Essa experiência é realizada em 64 horas divididas nos dois semestres. Para tanto, os alunos são divididos em grupos e, semanalmente, há revezamento do aluno que frequenta a unidade, passando todo o conteúdo da semana anterior para o próximo aluno, visando a dar continuidade ao trabalho que o grupo desenvolve na unidade.

O programa é desenvolvido de forma integral (8 horas/ dia) em quatro dias consecutivos da semana (terça a sexta-feira). O aluno da graduação ( $4^{\circ}$ ano) fica inserido nas atividades da USF durante uma semana e na clínica extramuros (clínica de Odontopediatria e CEO) da Faculdade de Odontologia de Piracicaba por mais uma semana. Isso ocorre no primeiro semestre, sendo que o mesmo processo acontece também no segundo semestre, totalizando quatro semanas de estágio no ano.

Na clínica de Odontopediatria, os alunos atendem crianças provenientes de escolas públicas municipais de Piracicaba, sempre orientados e supervisionados por professores e profissionais do serviço. Além do atendimento curativo, em parte da semana os alunos vão às escolas e realizam levantamento epidemiológi$\mathrm{co}$, classificando as crianças de acordo com risco e necessidade de tratamento12 e realizando escovaçãosupervisionada com gel fluoretado,conformeo risco de agravos odontológico que elas apresentam, e a escovação supervisionada com dentifríciofluoretado nas demais crianças. O aproveitamento da área escolar/ social como espaço clínico foi um importante avanço dos projetos, pois resultou no atendimento de crianças que necessitavam de procedimentos de controle da cárie dentária de baixa complexidade, que se fossem executados em ambiente clínico, iriam aumentar o tempo fora da sala de aula e, adicionalmente, ocupariam uma consulta em detrimento de outra criança que apresentasse necessidade clínica restauradora.

Essa atividade é muito importante, pois o aluno tem a oportunidade de conhecer a comunidade por meio de ações de planejamento em saúde e otimizar as ações de cuidados básicos ao utilizar os espaços sociais como espaço clínico.

Na Unidade de Saúde da Família são realizadas atividades de acordo com o planejamento local de cada Unidade, sendo, portanto, diversificado entre os grupos. O quadro 1 exemplifica um planejamento das atividades dos alunos em uma USF.

\section{CONSIDERAÇÕES FINAIS}

"Como a maioria dos alunos, para não dizer todos, fui ao estágio imaginando que iria porque não tinha escolha, porém, dia a dia minha 


\begin{tabular}{|c|c|c|c|c|}
\hline \multicolumn{5}{|c|}{ QUADRO 1} \\
\hline Período & terça-feira & quarta-feira & quinta-feira & sexta-feira \\
\hline $\begin{array}{l}\text { Manhã e/ou } \\
\text { tarde }\end{array}$ & $\begin{array}{l}\text { Atividades de clínica } \\
\text { no consultório e visitas } \\
\text { domiciliares. }\end{array}$ & $\begin{array}{l}\text { Toda quarta: Oficina de Arte } \\
\text { e Puericultura - conjunto de } \\
\text { noções e técnicas voltadas para } \\
\text { o cuidado médico, higiênico, } \\
\text { nutricional, psicológico etc. } \\
\text { das crianças pequenas, da } \\
\text { gestação até } 4 \text { ou } 5 \text { anos de } \\
\text { idade. } \\
\text { Atividade em espaço social. }\end{array}$ & $\begin{array}{l}\text { Grupo hipertenso e diabético } \\
\text { no período da manhã. } \\
\text { Visitas domiciliares e ações } \\
\text { de educação e prevenção em } \\
\text { saúde emescola municipal e } \\
\text { creche. }\end{array}$ & $\begin{array}{l}\text { Atendimento clínico a paciente } \\
\text { adulto, acolhimento e reunião } \\
\text { com a equipe da USF. }\end{array}$ \\
\hline Objetivos & $\begin{array}{l}\text { 1) Realizar visitas domiciliares } \\
\text { com ACS, tabular os dados } \\
\text { e priorizar as ações em } \\
\text { domicílio (prev., assistenciais e } \\
\text { educativas) e assistenciais em } \\
\text { clínica. } \\
\text { 2) Realizar e observar, a } \\
\text { critério do cirurgião-dentista, } \\
\text { atividades clínicas. }\end{array}$ & $\begin{array}{l}\text { Atividades em espaço social. } \\
\text { 1) Realizar levantamento } \\
\text { epidemiológico, priorizar } \\
\text { as ações em espaço escolar } \\
\text { (preventivas, educativas e } \\
\text { assistenciais: ART e flúor). } \\
\text { Organizar encaminhamento } \\
\text { para tratamento em clínica de } \\
\text { atenção primária e secundária. }\end{array}$ & $\begin{array}{l}\text { 1) Realizar visitas domiciliares } \\
\text { com ACS, tabular os dados } \\
\text { e priorizar as ações em } \\
\text { domicílio (prev., assistenciais e } \\
\text { educativas) e assistenciais em } \\
\text { clínica. } \\
\text { 2) Visita acompanhado da } \\
\text { enfermeira para observar } \\
\text { problemas de ordem } \\
\text { geral e encaminhar para o } \\
\text { cirurgião-dentista problemas } \\
\text { odontológicos. }\end{array}$ & $\begin{array}{l}\text { 1) Realizar e observar, a } \\
\text { critério do cirurgião-dentista, } \\
\text { atividades clínicas. } \\
\text { 2) Participar da reunião de } \\
\text { grupo e encaminhar para os } \\
\text { supervisores as sugestões. } \\
\text { 3) Planejar a próxima semana. }\end{array}$ \\
\hline
\end{tabular}

concepção foi mudando e me entreguei ao que viesse porque acreditava que tinha algum propósito nisso, não era à toa. Realmente estava enganada. Hoje sei que essa experiência foi e ainda será muito importante para a minha vida..."

(Relato da percepção de uma aluna a respeito do estágio.)

Essa atividade possibilita a experiência de praticar os conhecimentos obtidos na Faculdade, auxiliando significativamente na formação profissional do aluno. As atividades desenvolvidas na USF possibilitam ainda aos graduandos observar a dinâmica do trabalho no SUS e suas dificuldades, além de aprender a planejar ações de trabalho. Outro ponto importante é a interação das atividades desenvolvidas na USF com a pós-graduação, pois pós-graduandosacompanham as atividades dos graduandos e realizam pesquisas no âmbito do SUS.

A integração entre graduandos, pós-graduandos, docentes e equipe de saúde da família, proporcionada pelo Pró-Saúde em Piracicaba, foi positiva e enriquecedora para todas as partes envolvidas.

Outro aspecto importante é em relação à quebra de preconceitos relativos ao serviço público de saúde junto aos graduandos em Odontologia, dentre os quais foi possível romper com os seguintes paradigmas:

a. Emprego de procedimentos de baixa tecnologia não significa uma Odontologia de má qualidade.

b. Odontologia "social" não é sinônimo de uma Odontologia precária, sem qualidade e destinada a pessoas carentes ${ }^{13}$. c. O "postinho" do SUS não é para atendimento exclusivo de pessoas carentes. O SUS é para todos ${ }^{14}$.

d.Ser cirurgião-dentista generalista não é demérito nem motivo para vergonha; ser cirurgião-dentista generalista é necessário no Brasil, onde grande parte da população é SUS-dependente ${ }^{15}$.

Contudo, observa-se ainda que o grande obstáculo a ser superado pelo Pró-Saúde se encontra dentro da própria universidade enquanto instituição, mais especificamente, dentro do seu corpo docente, no qual o modelo biomédico de prática, resistente à capacidade de abstração, discussão, crítica e aprendizado de novos conceitos úteis para a prática clínica dos graduandos ainda é muito enraizado ${ }^{16,17}$.

\section{REFERÊNCIAS}

1. Brasil. Ministério da Saúde. Norma Operacional Básica do Sistema Único de Saúde (NOB-SUS 11/96). Brasília, 1996.

2. Souza RA, Carvalho AM. Programa de Saúde da Família e qualidade de vida: um olhar da Psicologia. Estud de Psicol 2003; 8(3), 515-523

3. Brasil. Ministério da Saúde, Portaria GM/MS nº 396, de 04 de abril de 2003, que reajusta incentivo financeiro para a reorganização da atenção à saúde bucal prestada nos municípios por meio do Programa de Saúde da Família pela Portaria GM/MS no 1.444 estabelece incentivo financeiro 
para a reorganização da atenção à saúde bucal prestada nos municípios por meio do Programa de Saúde da Família, de 28 de dezembro de 2000.

4. Manfredini, MA. Saúde bucal no programa saúde da família no Brasil. In: Dias, AA et al. Saúde Bucal Coletiva: Metodologia de Trabalho e Práticas. São Paulo: Santos; 2006

5. Narvai,PC. Saúde bucal: assistência ou atenção? São Paulo, mimeo, 1992.

6. Aerts D, Abegg C, Cesar K. O papel do cirurgião-dentista no Sistema Único de Saúde. Ciênc saúde coletiva 2004; 9(1): 131-38.

7. Pereira AC, Meneghim MC, Hebling, E. Extensão Universitária: Avaliação do estágio extra extra-muro desenvolvido pela FOP-UNICAMP. Rev Clínica Odontol 1995; 1(2): 16-20

8. Pereira AC, Meneghim MC, Gomes PR, Oliveira SP, Fortunato JC, Yassuhara AA. O Perfil Epidemiológico e sua relação com o planejamento de ações odontológicas no PIESE-Paulínia (SP). Saúde em Debate 1999; 23(53): 63-67.

9. Brasil. Ministério da Saúde. Ministério da Educação. Programa Nacional de Reorientação da Formação Profissional em Saúde - Pró-Saúde: objetivos, implementação e desenvolvimento potencial/Ministério da Saúde, Ministério da Educação. Brasília: Ministério da Saúde, 2005.

10. IBGE. Estimativas da população para $1^{\circ}$ de julho de 2008 (PDF). Instituto Brasileiro de Geografia e Estatística (IBGE) [on line]. 2008. [capturado 29 de agosto de 2008].

11. Meneguel L, Araújo MA, Del Castilho RAF. Introdução ao Tel Educ. In: Apostila do Tel Educ. Campinas: UNICAMP, 2005. 22p.

12. Nyvad B, Machiulskiene V, Baelum V. Reliability of a new caries diagnostic system differentiating between active and inactive caries lesions. Caries Res 1999; 33: 252-260.

13. Narvai PC. Odontologia e saúde bucal coletiva. São Paulo: Editora Hucitec; 2002.

14. Morita MC, Kriger L, Gasparetto A, Tanaka EE, Higasi MS, Mesas AE, Iwakura MLH, Alvanham D. Projeto Pró-Saúde
Odontologia: relato das atividades iniciais em Universidades do Estado do Paraná. Revista Espaço para a Saúde 2007; 8(2): 53-57.

15. Tarrío CM. Especialista ou clínico geral? Revista da APCD 1995; 49(1): 8-17.

16. Pereira AC. Operatória Dentária. In: Carlos Botazzo e Maria Aparecida de Oliveira. (Org.). Atenção básica no Sistema Único de Saúde: abordagem interdisciplinar para os serviços de saúde bucal. 1 ed. São Paulo: Páginas \& Letras Editora e Gráfica Ltda, 2008, v. 1, p. 173-180.

17. Matos M. formação de Cirurgião-Dentista e os desafios contemporâneos do mundo de trabalho [acesso em 26 de março de 2009]. Diponível em: http: //www.odontocloseup.com.br/web/artigo/FormacaoCirurgiao.aspx.

\section{CONTRIBUIÇÃO DOS AUTORES}

Marcelo de Castro Meneghim participou da concepção do projeto, do planejamento, da execução, da análise e rdação do artigo. Antonio Carlos Pereira participou da concepção do projeto, do planejamento, da análise e revisão da redação do artigo. Fábio Luiz Mialhe participou da concepção do projeto, do planejamento, da execução e revisão da redação do artigo. Maria da Luz R. De Sousa participou da concepção do projeto, do planejamento, da análise e revisão da redação do artigo.

\section{CONFLITO DE INTERESSES}

Declarou não haver.

\section{ENDEREÇO PARA CORRESPONDÊNCIA}

Marcelo de Castro Meneghim Faculdade de Odontologia de Piracicaba - Departamento de Odontologia Social — Unicamp

Avenida Limeira, 901

Bairro Areião - Piracicaba

CEP. 13414-901 SP

E-mail: meneghim@fop.unicamp.br 